\title{
Perfil clínico e epidemiológico das queimaduras: evidências para o cuidado de enfermagem
}

\section{Profile of clinical and epidemiological burns: evidence for nursing care}

\author{
Gabriela Oliveira Parentes da Costa ${ }^{a}$, Josué Alves da Silva ${ }^{b}$, Ariane Gomes dos Santos ${ }^{c}$ \\ a Enfermeira graduada pelo Instituto de Ensino Superior Múltiplo (IESM). \\ ${ }^{\mathrm{b}}$ Enfermeiro graduado pelo IESM. \\ c Enfermeira. Mestre em Enfermagem pela Universidade Federal do Piauí (UFPI). Professora do Curso de Enfermagem do IESM.
}

Objetivo: Analisar as evidências científicas disponíveis acerca dos cuidados de enfermagem a pacientes internados por queimaduras.

Materiais e Métodos: Trata-se de uma revisão integrativa da literatura. O período da coleta foi de setembro de 2014 a janeiro de 2015, por meio das bases de dados LILACS/SciELO; IBECS; MEDLINE e recorte temporal de 2002 a 2014. A amostra final foi de 18 artigos. Para avaliação da qualidade metodológica dos mesmos aplicou-se as recomendações STROBE (Strengthening the reporting of observational studies in epidemiology). Os dados foram analisados de forma descritiva.

Resultados: Para melhor organização os resultados foram agrupados em duas categorias analíticas; segundo as recomendações STROBE 55,5\% foram classificados como A e 44,4\% B; quanto ao nível de evidencia 72,2\% tiveram nível 4; 55,5\% dos estudos correspondem a publicações entre 2011 e 2014. Os indivíduos do sexo masculino foram os que mais se envolveram em queimaduras e as causas prevalentes foram contatos acidentais com líquidos quentes e manejo de álcool. A maioria ocorreu em ambiente doméstico, atingindo principalmente os membros superiores. No cotidiano os enfermeiros lidam com cuidados físicos e emocionais. Alguns profissionais relataram dificuldades em lidar com a dor do paciente, principalmente, durante o banho e curativo, procedimentos rotineiros na unidade de queimados.

Conclusão: Mesmo com as dificuldades enfrentadas a equipe de enfermagem deve sistematizar a assistência usando de artifícios científicos e tecnologias que contribuam para melhoria do cuidado prestado.

Palavras-chaves: queimaduras; cuidados de enfermagem; terapêutica; diagnóstico. through the LILACS / SCiELO databases; IBECS; MEDLINE and time frame of 2002 to 2014. The final sample of 18 articles. To assess the methodological quality of the applied the STROBE recommendations (Strengthening the reporting of observational studies in epidemiology). Data were analyzed descriptively.

Results: To better organize the results were grouped into two analytical categories; according to the recommendations STROBE 55.5\% were classified as A and $44.4 \%$ B; about the level of evidence $72.2 \%$ had level $4 ; 55.5 \%$ of the studies correspond to publications between 2011 and 2014. Males were the most engaged in burns and prevalent causes were accidental contact with hot liquids and management of alcohol. Most occurred in the home environment, mainly affecting the upper limbs. In everyday nurses deal with physical and emotional care. Some professionals reported difficulties in dealing with the patient's pain, especially during showering and dressing routine procedures in the burn unit.

Conclusion: Despite the difficulties facing the nursing team should systematize assistance using scientific devices and technologies that contribute to improve the care delivery.

Keywords: burns; nursing care; therapeutics; diagnosis.

\section{Correspondência:}

ARIANE GOMES DOS SANTOS

Residencial Portal do Cristo Rei, 1920, Bloco F, ap. 401

64015900 Teresina, PI, Brasil

E-mail: arianeg.santos@hotmail.com 


\section{INTRODUÇÃO}

Queimaduras são lesões dos tecidos orgânicos, ocasionadas por agentes químicos, físicos e biológicos. Podem ser classificadas quanto à profundidade como, primeiro grau, quando as lesões atingem somente a camada epidérmica; segundo grau, quando há comprometimento da epiderme e a camada superficial ou profunda da derme; e, terceiro grau acometendo, além da pele, outros tecidos como o subcutâneo, músculos, tendões e até mesmo os ossos. Quanto maior a área corporal queimada, maior o índice de mortalidade $^{1,2}$.

As causas mais frequentes das queimaduras são exposição ao fogo, água fervente, corrente elétrica, agentes químicos, solução cáustica, entre outros. O tipo de queimadura vai depender do comprometimento do tecido e de qual produto ou agente o indivíduo foi exposto. Para calcular a área de superfície corporal queimada (SCQ), são utilizadas com mais frequência a Regra dos Nove, em emergência e a tabela de Lund-Browder para queimaduras em crianças, por apresentar maior precisão em relação à proporção corporal e à idade ${ }^{3}$.

As queimaduras têm gerado gastos aos órgãos públicos e privados. A cada ano 2 milhões de pessoas sofrem queimaduras e o Sistema Único de Saúde- SUS gasta cerca de 55 milhões anualmente para o tratamento destes pacientes ${ }^{4}$.

Análises da morbidade hospitalar por causa externas no SUS, entre período de janeiro a junho de 2014, mostraram que, no Brasil, 6.782 pessoas sofreram lesões por fonte de calor, substâncias quentes, exposição à fumaça, ao fogo ou às chamas $^{5}$. Os principais fatores de riscos para estes acidentes são, manejo de álcool, festas com fogos de artifícios, fogueiras, balões, fatores socioeconômicos desfavoráveis e a violência ${ }^{6}$.

As crianças são as mais envolvidas em queimaduras, enquanto a população da terceira idade corresponde apenas a 10\% dos casos, porém, os idosos são os que permanecem por mais tempo hospitalizados devido às comorbidades da idade $^{7}$.

O tempo de internação do paciente que sofre algum tipo de queimadura pode ser prolongado, podendo resultar em estresse e sérios problemas biopsicossociais. Dependendo do grau da queimadura e da magnitude do estresse emocional, o paciente pode desenvolver estresse pós-traumático, sofrendo depressão, transtornos de personalidade e intelectuais e o abuso de substâncias psicoativas, que afetem as relações escolares, a interação familiar, conjugal e o trabalho ${ }^{8}$.

No Brasil, o número de Centro de Tratamento de Queimados é desproporcional à demanda. A complexidade no tratamento de queimados requer investimentos finan- ceiros, adequação da infraestrutura e equipe especializada e preparada9.

As vítimas de queimaduras podem apresentar necessidades de intervenções como, curativos, enxertos e retalhos; como consequência as cicatrizes podem desenvolver eritemas, discromias, hipertrofias e limitações de funcionalidade, necessitando de reavaliações de $\operatorname{condutas}^{10}$.

A principal característica da assistência da enfermagem é o cuidar e nesse contexto, a equipe deve estar preparada e habilitada para lidar com pacientes queimados. Estes necessitam de assistência que envolva aspectos físicos, emocionais e atenção à família, ou seja, uma assistência holística. O cuidado com a manutenção das vias aéreas, reposição de fluidos, controle da dor, a observação da atualização do calendário vacinal do paciente são de extrema importância. Além disso, cuidados com curativos, preservação de tecidos, prevenção e controle de infecções, visando à cicatrização da lesão em menor tempo possível, a fim de, evitar complicações, são algumas das atribuições da equipe de enfermagem ao paciente queimado ${ }^{11}$.

Para a realização desses cuidados essenciais e o alcance das metas traçadas, é necessário aplicar o processo de enfermagem (PE), uma vez que, este abrange o histórico do paciente, diagnósticos de enfermagem, planejamento da assistência prestada, além de implementação e avaliação dessas ações, possibilitando uma assistência organizada e resolutiva $^{12}$

Os diagnósticos de enfermagem (DE) compõem uma taxonomia única e permite uma comunicação padronizada de acordo com a North American Nursing Diagnosis Association (NANDA), efetivando a prática da assistência de enfermagem ${ }^{13}$

A realização desse estudo justifica-se pela necessidade de compreender o perfil de pacientes acometidos por queimaduras, bem como verificar como ocorre o trabalho da enfermagem diante dessa problemática, identificando as dificuldades encontradas na assistência ao binômio paciente e familiar. Tendo como objetivos: analisar as evidências científicas disponíveis acerca dos cuidados de enfermagem a pacientes internados por queimaduras; identificar o perfil clínico e epidemiológico de pacientes acometidos por queimaduras; identificar os principais desafios da equipe de enfermagem no cuidar do paciente queimado e evidenciar subsídios científicos que contribuam para assistência de enfermagem por meio da aplicabilidade do processo de enfermagem a pacientes queimados e familiares.

\section{MATERIAIS E MÉTODOS}

Trata-se de uma revisão integrativa da literatura com abordagem qualitativa. A revisão integrativa da literatura 
se embasa em estudos de relevância que possibilitem novos dados relacionados ao interesse da pesquisa, visando contextualizar o problema e contribuir para discussões e reflexões, proporcionando a construção do conhecimento ${ }^{14,15}$.

Por meio da abordagem qualitativa é possível entender a perspectiva dos participantes da amostra, possibilitando a interpretação e interação do pesquisador com o objeto de estudo $^{16}$.

Para direcionar esta pesquisa foram seguidas seis fases distintas: definição do tema e formulação dos objetivos e da questão norteadora; busca na literatura e delimitação para a inclusão dos estudos; categorização dos estudos; avaliação dos estudos; interpretação dos resultados; e apresentação da revisão/síntese do conhecimento ${ }^{14,17,18}$.

Este estudo foi guiado pela seguinte questão norteadora: Quais as evidências disponíveis sobre os cuidados de enfermagem a pacientes vítimas de queimaduras?

O período da coleta de dados foi de setembro 2014 a janeiro de 2015 na Biblioteca Virtual em Saúde - BVS, por meio das bases de dados Latino-Americana, Caribe em Ciências da Saúde - LILACS/ Scientific Eletronic Library Online - SciELO, Índice Bibliográfico Espanhol de Ciências da Saúde -IBECSe Literatura Internacional em Ciências da Saúde - MEDLINE.

O recorte temporal foi de 2000 a 2014. Para sistematizar a coleta da amostra foram utilizados o operador booleano "and" e os descritores controlados: queimaduras, enfermagem, terapêutica, assistência e diagnóstico, todos de acordo com BIREME, esquematizados conforme mostra a Figura1.

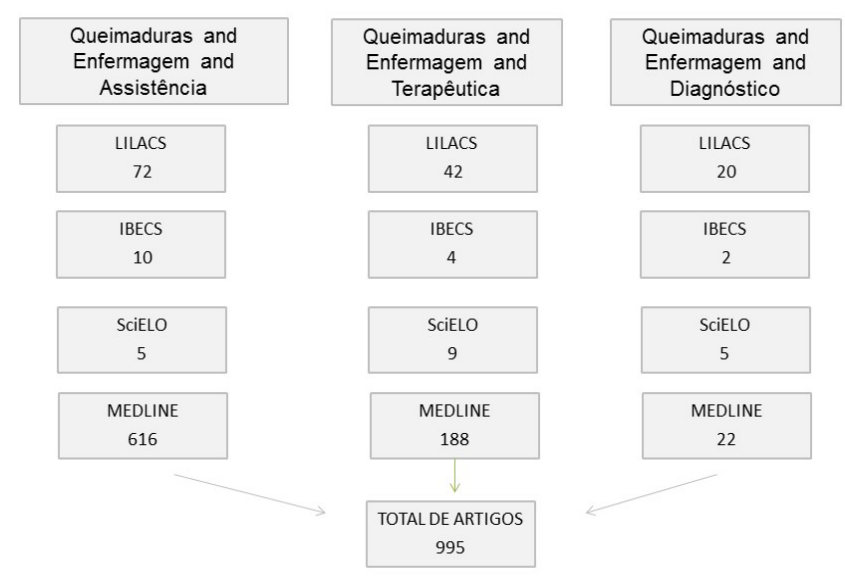

Figura 1. Estudos encontrados nas bases de dados.

A seleção da amostra iniciou com os critérios de inclusão, que abrangeram artigos publicados dentro do período de interesse da pesquisa, os que estavam com textos completos disponíveis na íntegra, estudos originais e que foram pertinentes ao presente estudo, nos idiomas português, inglês e espanhol. Já os critérios de exclusão consistiram em artigos repetidos, resenhas, anais de congresso, editoriais, artigos de revisão, estudos envolvendo animais, trabalhos de avaliação da qualidade metodológica Strengthening the reporting of observational studies in epidemiology (STROBE) com nota C, ou seja, que preenchiam menos que 50\% dos critérios de avaliação e artigos que não abordaram diretamente o tema deste estudo.

$\mathrm{Na}$ avaliação da qualidade metodológica dos estudos observacionais, aplicou-se as recomendações STROBE. Os 22 itens que compõem o STROBE foram pontuados de zero a um, de cada artigo, por dois autores, a soma da pontuação de cada um dos itens foi considerada então, a nota do estudo por cada autor. Ao final desse processo, a média da pontuação dos dois autores foi considerada para a nota final do estudo foram identificados pelas letras $\mathrm{A}$ os que obtiveram valor igual ou superior a $80 \%$; letra B os que apresentaram pontuação de 50 a $80 \%$ e letra $C$ os que obtiveram pontuação abaixo de $50 \%{ }^{19}$.

Foi realizada avaliação dos títulos e resumos o que possibilitou a exclusão de vários artigos. Após isso, foram selecionados 35 artigos os quais foram lidos e analisados na íntegra. Após avaliação pelo método STROBE, foram excluídos 17 artigos, por não se adequarem ao método de avaliação ou por pontuarem nota inferior a 50\% (C). Dessa forma, a amostra final do presente estudo constituiu-se de 18 artigos.

Para a coleta das informações dos estudos selecionados, foi utilizado um instrumento elaborado pelos autores que contemplou as seguintes variáveis: título, autores, periódico, ano de publicação, país de publicação, idioma, tipo de estudo, objetivos, sujeitos do estudo, amostra, resultados, conclusões, nível de evidência e valor do STROBE.

Após a seleção da amostra final os artigos foram classificados de acordo com o nível de evidência, da seguinte maneira: 1 - Metanálise de múltiplos estudos controlados; 2 - Estudo individual com delineamento experimental; 3 - Estudo com delineamento quase-experimental como estudo sem randomização com grupo único pré e pósteste, séries temporais ou caso-controle; 4 - Estudo com delineamento não-experimental como pesquisa descritiva correlacional e qualitativa ou estudos de caso; 5 - Relatório de casos ou dado obtido de forma sistemática, de qualidade verificável ou dados de avaliação de programas; e 6 - Opinião de autoridades respeitáveis baseada na competência clínica ou opinião de comitês de especialistas, incluindo interpretações de informações não baseadas em pesquisas $^{20}$. 
Os dados extraídos dos artigos selecionados foram analisados de forma descritiva, possibilitando observar, analisar e correlacionar os fatos com a maior precisão possíve ${ }^{21}$ e agrupados por semelhança de conteúdo em categorias analíticas.

\section{RESULTADOS E DISCUSSÃO}

A partir do instrumento de coleta de dados foi possível elaborar duas tabelas com a síntese dos artigos da amostra com os seguintes aspectos: autores e objetivo geral dos artigos (Tabela 1) e amostra, ano, desenho do estudo, nível de evidência e STROBE (Tabela2). Foram analisados na íntegra, 18 artigos que compuseram o escopo desta revisão.

Observou-se o predomínio de estudos que trataram da epidemiologia das queimaduras com 10 (55,6\%) dos artigos, seguidos de $6(33,3 \%)$ que se referiam ao processo de enfermagem no centro de tratamento de queimados, $2(11,1 \%)$ que citavam os desafios da enfermagem nessa temática.

Tabela 1. Caracterização dos estudos quanto aos autores e objetivo geral dos artigos.

\begin{tabular}{|c|c|}
\hline Autores & Objetivo geral dos artigos \\
\hline meneghetti et al. ${ }^{58}$ & Investigar os diagnósticos de enfermagem e sua relação com os cuidados prescritos \\
\hline Costa \& Rossi ${ }^{51}$ & Compreender os significados da atuação dos profissionais de enfermagem em uma Unidade de Queimados \\
\hline Martins \& Andrade 27 & Estudar a incidência hospitalar e a mortalidade por queimadura entre menores de 15 anos no município de Londrina \\
\hline Duarte et al. ${ }^{49}$ & Analisar a percepção dos profissionais de enfermagem sobre o seu trabalho em uma Unidade de Queimados \\
\hline Dutra et al. ${ }^{41}$ & Analisar as principais características de mulheres internadas por queimadura em um hospital no Rio de Janeiro \\
\hline Martins et al. ${ }^{50}$ & Desvelar os sentimentos vivenciados pela equipe de enfermagem que cuidam de pacientes com queimaduras \\
\hline Gawryszewski et al. ${ }^{25}$ & Descrever as características dos atendimentos decorrentes de queimaduras em serviços de urgência e emergência \\
\hline Calvet ${ }^{40}$ & Aumentar os conhecimentos sobre as principais características clínico e epidemiológicas decorrentes de acidentes por queimaduras \\
\hline Leão et al. ${ }^{24}$ & Divulgar o perfil epidemiológico das queimaduras em todo o estado de Minas Gerais \\
\hline Balan et al. ${ }^{22}$ & Estudar as características dos casos de queimaduras atendidos em uma unidade de emergência de um hospital-escola do Noroeste do Paraná \\
\hline Montes et al. ${ }^{23}$ & $\begin{array}{l}\text { Caracterizar os pacientes internados vítimas de queimaduras e identificar os tratamentos e procedimentos invasivos adotados e suas principais } \\
\text { complicações apresentadas }\end{array}$ \\
\hline Gonçalves et al. ${ }^{26}$ & $\begin{array}{l}\text { Descrever o perfil dos pacientes atendidos na Unidade de Queimados e identificar quais atitudes são realizados imediatamente após a } \\
\text { ocorrência da queimadura }\end{array}$ \\
\hline Biscegli et al. ${ }^{28}$ & Descrever o perfil de crianças e adolescentes vítimas de queimadura e comparar os resultados com dados existentes na literatura \\
\hline Millan et al. ${ }^{38}$ & Descrever a experiência da Unidade de Tratamento de Queimados do Serviço de Cirurgia Plástica do Hospital das Clínicas \\
\hline Dalri \& Carvalho ${ }^{55}$ & Avaliar o desempenho da aplicação de um software (PROGQUEM) para o planejamento da assistência de enfermagem \\
\hline Batista et al. ${ }^{60}$ & Investigar características clínicas de crianças vítimas de queimaduras e principais Diagnósticos de Enfermagem \\
\hline Goyotá et al. ${ }^{62}$ & Identificar os diagnósticos de enfermagem de familiares de pacientes queimados próximo à alta hospitalar \\
\hline Rossi $^{63}$ & Investigar os diagnósticos de enfermagem presentes em familiares de pacientes que sofreram queimaduras \\
\hline
\end{tabular}

Tabela 2. Caracterização dos estudos quanto à amostra, ano, desenho do estudo, nível de evidência e avaliação STROBE.

\begin{tabular}{|c|c|c|c|c|}
\hline Amostra & Ano & Desenho do estudo & Nível de evidência & Strobe \\
\hline 42 prontuários & 2005 & Transversal e retrospectivo & 4 & A \\
\hline 10 profissionais da equipe de enfermagem & 2003 & Transversal e descritivo & 4 & A \\
\hline 182 pacientes & 2007 & Transversal e descritivo & 4 & A \\
\hline 20 profissionaisda equipe de enfermagem & 2012 & Exploratório e descritivo & 4 & A \\
\hline 132 prontuários & 2011 & Retrospectivo de dados quantitativos & 4 & $\mathrm{~B}$ \\
\hline 20 profissionais da equipe de enfermagem & 2014 & Exploratório e descritivo & 4 & B \\
\hline 761 pacientes & 2012 & Transversal & 3 & A \\
\hline 790 pacientes & 2010 & Transversal e descritivo & 3 & B \\
\hline 687 pacientes & 2011 & Epidemiológico & 3 & $\mathrm{~B}$ \\
\hline 108 pacientes & 2009 & Descritivo e transversal & 4 & A \\
\hline 138 prontuários & 2011 & Retrospectivo, descritivo & 4 & A \\
\hline 211 pacientes & 2012 & Observacional e descritivo & 4 & A \\
\hline 382 prontuários & 2014 & Transversal e retrospectivo & 4 & A \\
\hline 129 crianças & 2012 & Epidemiológico & 3 & B \\
\hline 4 pacientes & 2002 & Estudo de caso & 5 & B \\
\hline 54 prontuários & 2011 & Documental, retrospectivo & 4 & B \\
\hline 10 familiares de pacientes & 2006 & Estudo de caso & 4 & A \\
\hline 17 familiares de pacientes & 2006 & Estudo de caso & 4 & B \\
\hline
\end{tabular}


Dos artigos avaliados por meio do STROBE, $10(55,6 \%)$ corresponderam à classificação A e $8(44,4 \%)$ tiveram classificação B. Quanto aos níveis de evidência, 13 estudos $(72,2 \%)$ obtiveram nível de evidência $4 ; 4$ estudos $(22,2 \%)$ obtiveram nível de evidência 3 e 1 estudo $(5,6 \%)$ teve nível de evidência 5. Em relação ao ano de publicação observou-se que $3(16,6 \%)$ se trataram de artigos publicados entre $2002 \mathrm{e}$ 2005; 5 (27,8\%) publicados entre 2006 e 2010 e $10(55,6 \%)$ entre 2011 e 2014.

Os resultados encontrados nas pesquisas foram distribuídos em duas categorias analíticas, a saber: "Perfil clínico e epidemiológico de pacientes acometidos por queimaduras" 10 (55,6\%) artigos e "Processo de Enfermagem na unidade de tratamento de queimados e os desafios para equipe de enfermagem" com 8 (44,4\%) artigos.

\section{Perfil clínico e epidemiológico de pacientes acometidos por queimaduras}

Identificar os aspectos epidemiológicos de qualquer doença é o passo inicial para elaboração de protocolos que melhorem a qualidade da assistência. No que se refere às queimaduras não é diferente. Vários estudos concluíram que os indivíduos do sexo masculino são os que mais se envolvem em queimaduras e as causas prevalentes são contatos acidentais com líquidos quentes e manejo de álcool e a maioria ocorreram em ambiente doméstico atingindo principalmente os membros superiores ${ }^{22-26}$

Em relação ao perfil epidemiológico das queimaduras em crianças e adolescentes dois estudos transversais com 182 e 382 pacientes, respectivamente, também encontraram os mesmos desfechos dos autores já citados, além da maior incidência em menores de 6 anos $^{27,28}$. Segundo a classificação, as queimaduras de $1 \underline{0}$ e $2 \underline{0}$ graus foram as mais prevalentes $22,23,28$.

O maior índice de queimaduras em homens deve-se ao fato de que eles costumam ser menos cautelosos e estão inseridos em um ambiente de trabalho insalubre e expostos muitas vezes a agentes inflamáveis sem nenhuma restrição $0^{4,29}$.

Deve haver uma interação entre as entidades trabalhistas e o Serviço Especializado em Engenharia de Segurança e Medicina no Trabalho - SESMT e a Comissão Interna de Prevenção de Acidentes - CIPA com o intuito de implantar estratégias para prevenção desses agravos. Prevenir é a melhor forma de evitar problemas à saúde ocupacional ${ }^{30}$.

O ambiente doméstico é o local onde ocorre a maioria das queimaduras, sendo a cozinha o compartimento da casa com maior índice dessas ocorrências, principalmente com líquidos superaquecidos e ocorrem geralmente ao se puxar panelas quentes sobre o fogão, mexer com lamparinas, velas entre outros. Esses acidentes comprometem, na maioria das vezes, os membros superiores ${ }^{31,32}$.

O álcool é um produto inflamável e de livre comercialização no Brasil. É utilizado para limpeza doméstica, combustão de lenha, carvão e armazenado em locais de fácil acesso. Este produto é a segunda substância com maior envolvimento em acidentes por queimaduras. Em 2002, a Agência Nacional de Vigilância Sanitária (ANVISA) por meio da Resolução no 46, proibiu a fabricação e a venda do álcool líquido a $96^{\circ} \mathrm{GL}$ (Gay-Lussac) no País, sendo permitida a venda em concentrações iguais ou menores que $54^{\circ} \mathrm{GL}$ e até o volume de 50 mililitros $^{33}$.

Os acidentes envolvendo substâncias inflamáveis no ambiente doméstico, muitas vezes, não decorrem da exposição às chamas, mas sim da natureza da substância que em contato com a pele e mucosas podem causar de leves a graves queimaduras. A livre comercialização de produtos inflamáveis como antissépticos e o armazenamento destes em casa expõem as pessoas, principalmente crianças, ao risco de sofrerem queimaduras.

Durante os 6 meses de proibição da venda do álcool houve a redução de $60 \%$ (90 mil adultos e 27 mil crianças) no número de acidentes com esse produto. Entretanto, uma liminar a favor de um grupo de fabricantes do produto foi concedida e os números de acidentes por queimaduras com esse produto voltaram a subir ${ }^{34-36}$.

Em vários países como Brasil, Reino Unido, China, Austrália e Canadá, as queimaduras estão entre as principais causas de acidentes envolvendo crianças. O que pode explicar o maior número do gênero masculino envolvido em queimaduras é o fato de que os meninos tendem a ter um comportamento mais ativo que as meninas ${ }^{37,38}$. As crianças com transtornos de atenção e hiperatividade, também têm maiores riscos de sofrerem queimaduras ${ }^{39}$.

Um estudo realizado em Cuba mostrou uma variação desse perfil, sendo as mulheres as mais atingidas ${ }^{40}$. No Brasil, as queimaduras por autoextermínio são mais prevalentes entre as mulheres ${ }^{24}$. Outro autor após realizar um levantamento retrospectivo de dados quantitativos em 132 prontuários de mulheres internadas por queimaduras pode verificar que a maior incidência foi na idade entre 13 e 43 anos e o agente mais utilizado para o feito foi o álcool ${ }^{41}$.

A tentativa de suicídio por queimaduras é um método de alta letalidade ${ }^{42}$. As tentativas de suicídio entre o sexo feminino, utilizando materiais inflamáveis, geralmente estão relacionadas com transtornos mentais sendo de três a 12 vezes maior do que em pacientes não psiquiátricos, sendo a depressão, os transtornos do humor e a esquizofrenia as 
principais causas ${ }^{43}$. As admissões por tentativa de suicídio, com fogo, chegam a $10 \%$ das internações e muitos dos casos são de tentativas repetidas ${ }^{8}$.

A forma usada para o suicídio depende da disponibilidade do método e de fatores culturais. Foi observado um índice elevado de autoextermínio com o uso de álcool e há uma ligação direta com a disponibilidade do produto no comércio $^{44,45}$. Os agentes inflamáveis estão entre as principais causas dos acidentes laborais, principalmente nas profissões mais insalubres como construção civil e industrial ${ }^{46}$.

Outro fator sobre o perfil epidemiológico dos pacientes queimados que merece ser evidenciado é a escolaridade. Estudo mostrou que a maioria dos pacientes tinham entre 9 e 11 anos de estudos, seguida dos que tinham entre 0 e 4 anos. Os menores índices foram entre os que possuíam 12 anos e mais de estudos ${ }^{25}$. Dessa forma, podese observar que a escolaridade pode ter influência direta sobre os altos índices de queimaduras, uma vez que, 90\% dos pacientes pesquisados possuíam baixo nível de escolaridade 47,48 .

\section{Processo de Enfermagem na unidade de tratamento de queimados e os desafios para equipe de enfermagem}

Um estudo em que buscou conhecer as perspectivas da equipe de enfermagem em cuidar de pacientes queimados evidenciou que os entrevistados sentem prazer em poder contribuir na evolução da melhora do quadro clinico do paciente e o êxito do empenho refletido no momento da alta hospitalar. Os desafios enfrentados pelos profissionais segundo o estudo foram: o choque inicial ao se deparar com o paciente, a dificuldade em lidar com o julgamento de culpa do familiar/mãe em relação a como ocorreu o evento da queimadura afetando a relação da equipe com o paciente e família, também citaram o despreparo para trabalhar com crianças, o convívio com o sofrimento do paciente e sua dor e a necessidade de maior preparo por parte das instituições de ensino na formação dos futuros profissionais para atuarem nessa área ${ }^{49}$.

Muitos profissionais possuem um sentimento de impotência diante da dor do paciente, além de estresse da equipe em lidar com essa situação. Para alguns profissionais o setor de queimados foi o mais difícil que tiveram que exercer durante a profissão. Além do estresse, relataram sentimento de impotência em lidar com a dor do paciente queimado e dificuldades em trabalhar com pacientes que já tentaram suicídio $^{50}$

No cotidiano os enfermeiros lidam com cuidados físicos e emocionais. Alguns profissionais relatam as dificuldades em lidar com a dor do paciente, principalmente, durante o banho e curativo, procedimentos rotineiros na unidade de queimados. É importante o olhar crítico da enfermagem no tocante as intervenções; um exemplo é o ajuste do horário das medicações para alívio da dor antes dos procedimentos mais incômodos para os pacientes ${ }^{51}$.

A equipe de enfermagem que atua em unidades de queimados são os profissionais que passam maior tempo com pacientes e devem estar preparados para atender às diversas situações. Em relação às dificuldades encontradas, lidar com a dor foi uma das situações mais estressantes para a equipe. Deve-se considerar que a dor é o $5^{\circ}$ sinal vital; ela é complexa, e deve ser tratada individualmente. Para isso, existem instrumentos como as escalas de avaliação da dor, as quais auxiliam na identificação da mesma e da sua intensidade, sendo que a equipe de enfermagem poderá utilizá-las melhorando as condições para realização das atividades, reduzindo o estresse tanto do paciente quanto da equipe. ${ }^{52}$.

Psicologicamente cada pessoa usa estratégias de enfrentamentos que vão desde fuga até a resolubilidade do problema. Os indicadores de estresse e enfrentamento, assim como altas demandas podem inferir no controle no trabalho ${ }^{53}$. Os profissionais devem controlar suas emoções, pois precisam ajudar os pacientes a lidar com esse momento de sofrimento ${ }^{54}$.

Além das dificuldades físicas e emocionais com as quais os enfermeiros têm que conviver, existem também as dificuldades em implementar a Sistematização da Assistência de Enfermagem (SAE). Isso ocorre, especialmente, devido ao tempo despendido para documentar manualmente as informações e à falta de recursos tecnológicos para ajudar nesse processo. Autores fundamentados cientificamente, implementaram um Software (PROGQUEM) para aplicação do PE em uma unidade de queimados, esse sistema mostrouse eficaz na organização das informações das etapas do processo, proporcionando agilidade e permitindo que o enfermeiro tenha mais tempo para cuidados diretos e individualizados aos pacientes ${ }^{55}$.

A inserção do prontuário eletrônico como um instrumento facilitador melhora a comunicação entre a equipe proporcionando rápida troca de informações sobre exames, necessidade de transferência internas, passagem de plantão, tomada de decisões e principalmente a otimização do tempo. Isso permite ao enfermeiro maior disponibilidade potencializando a resolutividade do cuidado $^{56}$.

A informatização de documentos do sistema de Assistência de Enfermagem, a partir dos diagnósticos de enfermagem, requer do enfermeiro a sensibilização para formulação de um documento formal com linguagem padronizada com base nos padrões internacionais como, por exemplo, a NANDA 
de forma informatizada para o levantamento de diagnósticos. Isso norteará a intervenção e a avaliação de enfermagem ao paciente como etapas fundamentais do $\mathrm{PE}^{56,57}$.

Outra etapa importante do PE é o plano de cuidados de enfermagem, que deve elencar todas as necessidades do paciente, além de estabelecer prioridades que permitam ao enfermeiro argumentar com o médico e demais componentes da equipe multiprofissional sobre mudanças na terapêutica conforme alterações no quadro de saúde do paciente. Conhecer suas necessidades só será possível por meio da comunicação efetiva e da realização de exames físicos bem feitos, atentando sempre ao fato de que o registro das informações no prontuário influenciará na continuidade desse processo ${ }^{3}$.

Um estudo retrospectivo realizado em uma Unidade de Queimados no estado de São Paulo buscou identificar a relação entre os DE e as prescrições de enfermagem, por meio da análise de 42 prontuários. O DE, para Integridade tissular prejudicada foi observado em todos os casos $(100 \%)$ e, Padrão respiratório ineficaz, Náusea e Distúrbio do padrão do sono, foram os menos diagnosticados, presentes em apenas em 03 dos casos $(7,1 \%)$. Em relação ao DE e prescrição dos cuidados, não havia divergência segundo a literatura, porém identificou-se que para uma assistência integral, tais prescrições se mostraram insuficientes no atendimento às reais necessidades dos pacientes sendo feita a sugestão de prescrições pelos autores, com base na literatura ${ }^{58}$.

$O$ profissional de enfermagem ao desenvolver uma assistência organizada através do PE, deverá ser capaz de correlacionar o saber multidisciplinar para o estabelecimento de relações de trabalho. Deve assegurar que as informações obtidas pelo instrumento de coleta de dados sejam completas, a fim de obter-se um senso padrão entre saúde e doença, pois além de possibilitar o levantamento dos diagnósticos de enfermagem, norteiam a dinâmica do serviço e assistência individualizada aos clientes assistidos ${ }^{59}$.

Os DE são individuais e devem identificar problemas reais e de risco, visando atender as necessidades do paciente. Um estudo documental identificou que em uma unidade de tratamento de queimados os DE prevalentes na amostra foram os do tipo Real: integridade tissular prejudicada; hipertermia relacionada ao trauma; ansiedade relacionada à crise situacional, e diagnóstico do tipo Risco: risco de disfunção neurovascular periférica; risco de infecção e risco de desequilíbrio na temperatura corporal. Em relação com a situação, o diagnóstico de ansiedade foi um dos mais frequentes nos registros de enfermagem, provavelmente por ser um fator diretamente relacionado à dor do paciente ${ }^{60}$.

O levantamento dos diagnósticos de enfermagem pelo enfermeiro exige amplo reconhecimento sobre diversos problemas e situações que afetam o processo saúdedoença do paciente e do familiar envolvido no processo de recuperação e reabilitação da saúde. Dessa forma, é de fundamental importância a qualificação do enfermeiro para assistência a pacientes com queimaduras, para a correta identificação dos problemas e elaboração de prescrições individualizadas. Além disso, o enfermeiro poderá incentivar a equipe para a melhoria da execução das atividades da enfermagem.

O enfermeiro deve possuir um pensamento crítico para planejar a assistência de forma que atenda integralmente e holisticamente as reais necessidades do paciente e optar pela melhor conduta possível. Não basta prescrever cuidados, o enfermeiro deve certificar-se de que as respostas a estas prescrições estão sendo positivas. Identificar o diagnóstico possibilita melhor planejamento dessa assistência ${ }^{12,61}$.

O cuidado de enfermagem como um processo assistencial integral deve abranger também os familiares de pacientes internados. Para estes, encontrou-se os diagnósticos do tipo real: conhecimento deficiente; ansiedade; disposição para enfrentamento familiar aumentado; padrão de sono perturbado e tensão devida ao papel de cuidador, em um período próximo à alta hospitalar ${ }^{62}$.

Em um estudo com 17 membros de famílias de pacientes queimados evidenciou-se dezoito diagnósticos de enfermagem, dos quais sofrimento de pesar antecipado, desempenho de papéis (mãe e pai) ineficaz e ansiedade foram os principais do tipo real identificados e os de risco foram: risco para violência direcionada a outros, risco para violência direcionada a si, risco para maternidade e paternidade prejudicada e risco para tensão aumentada devida ao papel de cuidador. Em um dos casos houve a necessidade de denúncia de maus tratos da mãe por parte da equipe e o mesmo foi encaminhado ao Conselho Tutelar ${ }^{63}$.

A internação hospitalar como consequência do acometimento físico por queimaduras causa, também, um abalo emocional para o paciente e seu familiar. Nesse contexto, o cuidado de enfermagem com uma abordagem integral permitirá realizar as intervenções direcionadas a ambos com propósito de lhes trazer conforto e preservação da qualidade de vida ${ }^{64}$.

A complexidade na assistência às crianças exige da enfermagem abrangência de conhecimentos técnicos e científicos, a fim de diminuir a morbidade e todas as complicações físicas e psicológicas do paciente queimado e seu familiar. Com base nisso, em uma unidade de tratamento de queimados no estado de São Paulo foi suspensa a internação de crianças vítimas de queimaduras até a qualificação da equipe de enfermagem para atendimento a esse público ${ }^{65}$. 
A qualificação profissional para atuação em Centro de Tratamento de Queimados é bastante importante para o enfrentamento das mais variadas realidades existentes, que podem interferir na recuperação do doente. No entanto, nota-se a escassez de recursos humanos qualificados, tecnológicos e de materiais para a realização da assistência e reabilitação de vítimas de queimaduras. Assim, o convívio com o sofrimento do paciente e familiar e as dificuldades de implantar a sistematização da assistência de enfermagem são grandes desafios para enfermeiros que atuam unidades de queimados.

Por meio das pesquisas realizadas para elaboração desta revisão, foi possível observar que os fatores que causam queimaduras são, na maioria das vezes, evitáveis e que o aumento crescente das estatísticas sobre queimaduras a tornam um grave problema de saúde pública. Dessa forma, torna-se importante a realização de educação em saúde nas escolas, hospitais, unidades básicas de saúde e na própria comunidade, a fim de evitar acidentes domésticos que culminem em queimaduras, isso contribuirá para a diminuição da morbimortalidade causada por esse agravo.

O presente estudo mostrou a grande relevância da assistência de enfermagem holística a pacientes queimados. Pôde-se perceber que o desgaste da equipe de saúde e a falta de recursos humanos e tecnológicos em Centros de Tratamento de Queimados causam grandes impactos na qualidade da assistência prestada. Torna-se necessária a busca de ferramentas eficazes para melhoria da qualidade de assistência prestada ao paciente e familiar.

Chama-se a atenção dos gestores públicos sobre a facilidade de se adquirir o álcool, o agente inflamável com maior envolvimento em queimaduras. Deve-se restringir a venda desse produto e realizar campanhas de conscientização a população sobre os cuidados com o seu uso. Além disso, deve haver medidas de prevenção de acidentes em ambiente doméstico e de trabalho, como a fiscalização dos materiais inflamáveis e acondicionamento adequado, visto que esses foram os locais onde ocorreram maior incidência de queimaduras. Outra medida eficaz seria a realização de campanhas divulgadas na mídia, com orientações sobre cuidados para se evitar acidentes por queimaduras.

Torna-se importante a realização de trabalhos educativos sobre o tema em escolas já que as crianças e adolescentes também fazem parte do perfil epidemiológico das queimaduras. A equipe de enfermagem que atua no centro de tratamento de queimados também pode ser mediadora do conhecimento participando da orientação aos familiares de pacientes internados.

Houve uma limitação em relação à amostra para realização desta revisão, uma vez que a maioria dos artigos primários já publicados sobre a temática foram realizados nas décadas de 80 e 90. No entanto, essa limitação não diminuiu a relevância do presente estudo, já que, todos os estudos selecionados apresentaram grande relevância, bons níveis de evidência e permitiram que todos os objetivos do presente estudo fossem alcançados.

Esse estudo mostrou um avanço nas publicações entre os anos de 2011 a 2014, vislumbrando, assim, a elevação do interesse pela área em questão. Porém, ainda é necessária a realização de mais estudos para fortalecer as evidências sobre a assistência de enfermagem a pacientes queimados.

\section{REFERÊNCIAS}

1. Almeida JWF, Santos JN. Assistência de enfermagem em grupos de riscos a queimadura. Rev Bras Queimaduras. 2013;12(2):71-6.

2. Lima OBA, Arruda AJCG, Carvalho GDA, Melo VC, Silva AF. A enfermagem e o cuidado à vítima de queimaduras: revisão integrativa. Rev Enferm UFPE. 2013;(7 nesp):4944-50. http:// dx.doi.org/10.5205/reuol.4700-39563-1-ED.0707esp201316

3. Oliveira TS, Moreira KFA, Gonçalves TA. Assistência de enfermagem com pacientes queimados. Rev Bras Queimaduras. 2012;11(1)31-7.

4. Macedo AC, Proto RS, Moreira SS, Gonella HA. Estudo epidemiológico dos pacientes internados na Unidade de Tratamento de Queimados do Conjunto Hospitalar de Sorocaba entre 2001 a 2008. Rev Bras Queimaduras. 2012;11(1):23-5.

5. Ministério da Saúde (BR). DATASUS. Informações de Saúde (TABNET). Epidemiológicas e Morbidades. Internações segundo região. [online]. Brasília: Ministério da Saúde; 2014.

6. Castro ANP, Silva DMA, Vasconcelos VM, Lima Júnior EM, Camurça MNS, Martins MC. Sentimentos e dúvidas do paciente queimado em uma unidade de referência em Fortaleza - CE. Rev Bras Queimaduras. 2013;12(3):159-64.

7. Nascimento LKA, Barreto JM, Costa ACSM. Análise das variáveis grau e porte da queimadura, tempo de internação hospitalar e ocorrência de óbito em pacientes admitidos em uma Unidade de Tratamento de Queimados. Rev Bras Queimaduras. 2013; 12(4):256-9.

8. Laporte GA, Leonardi DF. Transtorno de estresse pós-traumático em pacientes com sequelas de queimaduras. Rev Bras de Queimaduras. 2010;9(3):105-14.

9. Henrique DM, Silva LD, Costa ACR, Rezende APMB, Santos JAS, Menezes MM, Maurer TC. Controle de infecção no centro de tratamento de queimados: revisão de literatura. Rev Bras Queimaduras. 2013;12(4):230-4.

10. Gonçalves AC, Gonçalves N, Catapani LB, Rossi LA, Guirro ECO, Farina Júnior JA. Avaliação de diferentes áreas de cicatriz na vítima de queimadura pela utilização do Cutometer: relato de um caso. Rev Bras Queimaduras. 2013;12(4):289-92.

11. Rossi LA, Menezez MAJ, Gonçalves N, Ciofi-Silva CL, Farina Junior JA, Stuchi RAG. Cuidados locais com as feridas das queimaduras. Rev Bras Queimaduras. 2010;9(2):54-9.

12. Silva RMA, Castilhos APL. A identificação de diagnósticos de enfermagem em paciente considerado grande queimado: um facilitador para implementação das ações de enfermagem. Rev Bras Queimaduras. 2010;9(2):60-5. 
13. Barichello E, Silva MCV, Barbosa MH, Iwamoto HH. Diagnósticos de enfermagem em pacientes internados por queimaduras. Enfermería Global. 2010;9(3):1-8.

14. Mendes KDS, Silveira RCCP, Galvão CM. Revisão integrativa: método de pesquisa para a incorporação de evidências na saúde e na enfermagem. Texto Contexto Enferm. 2008;17(4):758-64. http:// dx.doi.org/10.1590/S0104-07072008000400018

15. Crossetti MGO. Revisão integrativa de pesquisa na enfermagem o rigor cientifico que Ihe é exigido. Rev Gaúcha Enferm. 2012; 33(2):8-9.

16. Medeiros M. Pesquisas de abordagem qualitativa. Rev Eletr Enfermagem. 2014;14(2):224-5.

17. Pompeo DA, Rossi LA, Galvão CM Revisão integrativa: etapa inicial do processo de validação de diagnóstico de enfermagem. Acta Paul Enferm. 2009;22(4):434-8. http://dx.doi.org/10.1590/S010321002009000400014

18. Souza MT, Silva MD, Carvalho R. Revisão integrativa: o que é e como fazer. Einstein. 2010;8(1):102-6. http://dx.doi.org/10.1590/ S1679-45082010RW1134

19. Elm EV, Altman DG, Egger M, Pocock SJ, Gotzsche PC, Vandenbroucke JP. The Strengthening the Re-porting of Observational Studies in Epidemiology (STROBE) Statement: Guidelines for Reporting Observational Studies. J Clin Epidemiol. 2008;61(4):344-9. http:// dx.doi.org/10.1016/j.jclinepi.2007.11.008

20. Oliveira YCA, Coura AS, Costa GMC, França ISXL. Comunicação entre profissionais de saúde-pessoas surdas: revisão integrativa. Rev enferm UFPE. 2015;9(2):957-64.

21. Cervo AL, Bervian PA, Silva R. Metodologia cientifica. 6 ${ }^{\underline{a}}$ ed. São Paulo: Pearson Prentice Hall; 2007. 162 p.

22. Balan MAJ, Oliveira MLF, Trassi G. Características das vítimas de queimaduras atendidas em unidade de emergência de um hospital escola do noroeste do Paraná. Ciênc Cuid Saúd. 2009;8(2):169-75. http://dx.doi.org/10.4025/cienccuidsaude.v8i2.8195

23. Montes SF, Barbosa MH, Sousa Neto ALS. Aspectos clínicos e epidemiológicos de pacientes queimados internados em um Hospital de Ensino. Rev Esc Enferm USP. 2011;45(2):369-73. http:// dx.doi.org/10.1590/S0080-62342011000200010

24. Leão CEG, Andrade ES, Fabrini DS, Oliveira RA, Machado GLB, Gontijo LC. Epidemiologia das queimaduras no estado de Minas Gerais. Rev Bras Cirur Plást. 2011;26(4):573-7.

25. Gawryszewski VP, Bernal RTI, Silva NN, Morais Neto OL, Silva MMA, Mascarenhas MDM, Sá NNB, Monteiro RA, Malta DC. Atendimentos decorrentes de queimaduras em serviços públicos de emergência no Brasil, 2009. Cad Saúde Pública. 2012; 28(4):629-40. http://dx.doi.org/10.1590/S0102-311X20120004 00003

26. Gonçalves AC, Echevarría-Guanilo ME, Gonçalves N, Rossi LA, Farina Junior JA. Caracterização de pacientes atendidos em um serviço de queimados e atitudes no momento do acidente. Rev Eletr Enferm. 2012;14(4):866-72. http://dx.doi.org/10.5216/ree. v14i4.15186

27. Martins CBG, Andrade SM. Queimaduras em crianças e adolescentes: análise da morbidade hospitalar e mortalidade. Acta Paul Enferm. 2007;20(4):464-9. http://dx.doi.org/10.1590/S010321002007000400013
28. Biscegli TS, Benati LD, Faria RS, Boeira TR, Cid FB, Gonsaga RAT. Perfil de crianças e adolescentes internados em Unidade de Tratamento de Queimados do interior do estado de São Paulo. Rev Paul Pediat. 2014;32(3):177-82. http://dx.doi.org/10.1590/01030582201432305

29. Reis IF, Moreira CA, Costa ACSM. Estudo epidemiológico de pacientes internados na unidade de tratamento de queimados do hospital de urgência de Sergipe. Rev Bras Queimaduras. 2011; 10(4):114-8.

30. Silva LS, Valente GSC. Riscos químicos hospitalares e gerenciamento dos agravos à saúde do trabalhador de enfermagem. Rev Pesq Cuid Fundam. 2012;(Supl.):21-4.

31. Cruz BF, Cordovil PBL, Batista KANM. Perfil epidemiológico de pacientes que sofreram queimaduras no Brasil: revisão de literatura. Rev Bras Queimaduras. 2015;11(4):246-50.

32. Oliveira ADS, Carvalho JR, Carvalho MS, Landim RSMP. Perfil das crianças vítimas de queimaduras atendidas em hospital público de Teresina. Rev Interd. 2013;6(2):8-14.

33. Agência Nacional de Vigilância Sanitária. Álcool gel reduz acidentes em 60\%. [online]. Brasília: ANVISA; 2004.

34. Agência Nacional de Vigilância Sanitária. Res. no 46 de 20 de fevereiro de 2002 [online]. Brasília: ANVISA; 2002.

35. Medeiros ACS, Albuquerque BCH, Mignoni ISP, Pereima MJL, Baungratz MM, Feijó RS. Análise das causas de morte em uma unidade de queimados do Hospital Infantil Joana de Gusmão (HIJG), de janeiro de 1991 a dezembro de 2012. Rev Bras Queimaduras. 2013;12(3):153-8.

36. Tíbola J, Barbosa E, Renck LI, Guimarães FSV, Kroeff MS, Pereima MJL. The liqüid alcohol in Brazilian current context. Burns. 2007; 33(3):19. http://dx.doi.org/10.1016/j.burns.2006.10.048

37. Rocha HJS, Lira SVG, Abreu RNDC, Xavier EP, Vieira LJES. Perfil dos acidentes por líquidos aquecidos em crianças atendidas em centro de referência de Fortaleza. Rev Bras Prom Saúde. 2007;20(2): 86-91. http://dx.doi.org/10.5020/18061230.2007.p86

38. Millan LS, Gemperli R, Tovo FM, Mendaçolli TJ, Gomez DS, Ferreira MC. Estudo epidemiológico de queimaduras em crianças atendidas em hospital terciário na cidade de São Paulo. Rev Bras Cir Plást. 2012;27(4):611-5. http://dx.doi.org/10.1590/S198351752012000400024

39. Badger KL, Anderson RJ, Kagan RJ. Attention deficit-hyperactivity disorder in children with burn injuries. J Burn Care Res. 2008; 29(5):724-9. http://dx.doi.org/10.1097/BCR.0b013e31818480e1

40. Calvet MAM. Características clinico epidemiológicas de los accidentes por quemaduras en el municipio de Contramaestre. Medisan. 2010;14(3):31.

41. Dutra AS, Penna LHG, Vargens OMC, Serra MCVF. Caracterização de mulheres hospitalizadas por queimaduras. Rev Enferm UER]; 2011;19(1):34-9.

42. Brito MEM, Goes LSP, Costa VB, Gurgel MGI, Alves MDS, Timbó MA, Filho JGB. Tentativa de suicídio por queimadura: ideação suicida e desesperança. Rev Bras Queimaduras. 2013;12(1):30-6.

43. Carvalho ID, Freitas MCV, Macieira L. Tentativa de autoextermínio com queimaduras - CTQ-HFA-RJ. Rev Bras Queimaduras. 2014; 13(2):95-8 
44. Macedo JLS, Rosa SC, Silva MG. Queimaduras autoinfligidas: tentativa de suicídio. Rev Col Bras Cir. 2011;38(6):387-91.

45. Aldunate JLCB, Ferrari Neto O, Tartare A, Araujo CAL, Silva CC, Menezes MAJ, Gomez DS, Ferreira MC. Análise de 10 anos de casos de queimaduras por álcool com necessidade de internação em hospital quaternário. Rev Bras Queimaduras. 2012; 11(4):220-5.

46. Nestor A, Turra K. Perfil epidemiológico dos pacientes internados vítimas de queimaduras por agentes inflamáveis. Rev Bras Queimaduras. 2014;13(1):44-50.

47. Albuquerque MLL, Silva GPF, Diniz DMSM, Figueiredo AMF, Câmara TMS, Bastos VPD. Análise dos pacientes queimados com sequelas motoras em um hospital de referência na cidade de Fortaleza-CE. Rev Bras Queimaduras. 2010;9(3):89-94.

48. Guimarães IBA, Martins ABT. Qualidade de vida de pacientes com queimaduras internados em um hospital de referência no nordeste brasileiro. Rev Bras Queimaduras. 2013;12(2):103-7.

49. Duarte MLC, Lemos L, Zanini LN, Wagnes ZI. Percepções da equipe de enfermagem sobre seu trabalho em uma unidade de queimados. Rev Gaúcha Enferm. 2012;33(1):77-84.

50. Martins JT, Bobroff MCC, Ribeiro RP, Soares MH, Robazzi MLC, Marziale MHP. Sentimentos vivenciados pela equipe de enfermagem de um centro de tratamento de queimados. Esc Anna Nery; 2014;18(3):522-6.

51. Costa ECFB, Rossi LA. As dimensões do cuidado em uma unidade de queimados: um estudo etnográfico. Rev Esc Enferm USP. 2003;37(3):72-81. http://dx.doi.org/10.1590/S0080-62342 003000300009

52. Silva BA, Ribeiro FA. Participação da equipe de enfermagem na assistência à dor do paciente queimado. Rev Dor. 2011;12(4):342-8. http://dx.doi.org/10.1590/S1806-00132011000400011

53. Negromonte MRO, Araujo TCCF. Impacto do manejo clínico da dor: avaliação de estresse e enfrentamento entre profissionais de saúde. Rev Latino-Am Enferm. 2011;19(2):1-7.

54. Pinto E, Della-Flóra AM, Silva LD, Rorato TJ, Requia J, Martins ESR, Zamberlan C, Marinho MGR. O sentimento e a assistência de enfermagem perante um grande queimado. Rev Bras Queimaduras. 2014;13(3):127-9.

55. Dalri MCB, Carvalho EC. Planejamento da assistência de enfermagem a pacientes portadores de queimadura utilizando um software: aplicação em quatro pacientes. Rev Latino-Am Enferm.
2002; 10(6):787-93. http://dx.doi.org/10.1590/S0104-11692002 000600006

56. Ribeiro JC, Ruoff AB, Baptista CLB. Informatização da Sistematização da Assistência de Enfermagem: avanços na gestão do cuidado. J Health Inform. 2014;6(3):75-80.

57. Labbadia LL, D'Innocenzo M, Fogliano RRF, Silva GEF, Queiroz RMRM, Carmagnani MIS, Salvador ME. Sistema Informatizado para Gerenciamento de Indicadores da Assistência de Enfermagem do Hospital São Paulo. Rev Esc Enferm USP. 2011;45(4):1013-37. http://dx.doi.org/10.1590/S0080-62342011000400032

58. Meneghetti RAS, Rossi LA, Barruffini RCP, Dalri MCB, Ferreira E. Planejamento da assistência a pacientes vítimas de queimaduras: relação entre os problemas registrados e cuidados prescritos. Rev Esc Enferm USP. 2005;39(3):268-79. http://dx.doi.org/10.1590/S008062342005000300004

59. Bittar DB, Pereira LV, Lemos RC. A Sistematização da assistência de enfermagem ao paciente crítico: proposta de instrumento de coleta de dados. Texto Contexto Enferm. 2006;15(4):617-28. http:// dx.doi.org/10.1590/S0104-07072006000400010

60. Batista LTO, Rodrigues FA, Vasconcelos JMB. Características clínicas e diagnósticos de enfermagem em crianças vítimas de queimadura. Rev Rene. 2011;12(1):158-65.

61. Canela AF, Sória DAC, Barros FE, Melos ROL, Castro RC. Monitorização do paciente grande queimado e as implicações na assistência de enfermagem: relato de experiência. Rev Bras Queimaduras. 2011;10(4):133-7.

62. Goyotá SLT, Rossi LA, Dalri MCB. Diagnósticos de enfermagem de familiares de pacientes adultos queimados no período próximo à alta hospitalar. Rev Latino-am Enferm. 2006;14(1):102-9. http:// dx.doi.org/10.1590/S0104-11692006000100014

63. Rossi LA. Diagnósticos de enfermagem presentes em familiares de pacientes vítimas de queimaduras. Rev Esc Enferm USP. 2006;40(3):356-64. http://dx.doi.org/10.1590/S0080-62342006 000300007

64. Nishi PK, Costa ECNF. Cuidados de enfermagem à pacientes vítimas de queimaduras: identificação e características clínicas. Revista UNINGÁ. 2013;14(1):181-92.

65. Lacerda LA, Carneiro AC, Oliveira AF, Gragnani A, Ferreira LM. Estudo epidemiológico da Unidade de Tratamento de Queimaduras da Universidade Federal de São Paulo. Rev Bras Queimaduras. 2010;9(3):82-8. 